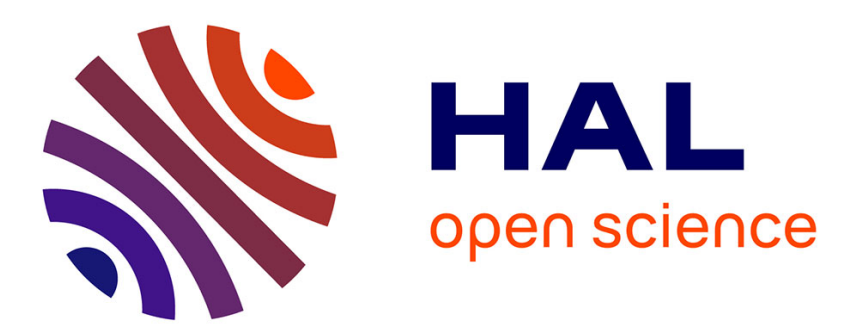

\title{
Première observation de la structure hyperfine magnétique dans le spectre infrarouge de l'ammoniac
}

Ch. Salomon, Ch. Chardonnet, A. van Lerberghe, Ch. Bréant, Ch. J. Bordé

\section{To cite this version:}

Ch. Salomon, Ch. Chardonnet, A. van Lerberghe, Ch. Bréant, Ch. J. Bordé. Première observation de la structure hyperfine magnétique dans le spectre infrarouge de l'ammoniac. Journal de Physique Lettres, 1984, 45 (23), pp.1125-1129. 10.1051/jphyslet:0198400450230112500 . jpa-00232459

\section{HAL Id: jpa-00232459 https://hal.science/jpa-00232459}

Submitted on 1 Jan 1984

HAL is a multi-disciplinary open access archive for the deposit and dissemination of scientific research documents, whether they are published or not. The documents may come from teaching and research institutions in France or abroad, or from public or private research centers.
L'archive ouverte pluridisciplinaire HAL, est destinée au dépôt et à la diffusion de documents scientifiques de niveau recherche, publiés ou non, émanant des établissements d'enseignement et de recherche français ou étrangers, des laboratoires publics ou privés. 
Classification

Physics Abstracts

$33.20 \mathrm{E}-07.65 \mathrm{G}$

\title{
Première observation de la structure hyperfine magnétique dans le spectre infrarouge de l'ammoniac
}

\author{
Ch. Salomon, Ch. Chardonnet, A. Van Lerberghe, Ch. Bréant et Ch. J. Bordé \\ Laboratoire de Physique des Lasers $(*)$, Université Paris-Nord, \\ avenue J.-B. Clément, 93430 Villetaneuse, France
}

(Reçu le 7 août 1984, accepté le 2 octobre 1984)

\begin{abstract}
Résumé. - Nous présentons la première observation expérimentale, par spectroscopie de saturation, de la structure hyperfine magnétique dans le spectre infrarouge de l'ammoniac. Le spectre de la transition as $\mathrm{R}(2.0)$ de la bande $v_{2}$ de ${ }^{15} \mathrm{NH}_{3}$. en quasi-coïncidence avec la raie $10 \mathrm{R}(42)$ du laser à $\mathrm{CO}_{2}$, a été enregistré avec une demi-largeur de raie de $2 \mathrm{kHz}$, limitée par le temps de vol des molécules à travers le faisceau laser. Ce spectre s'est avéré très sensible à un champ magnétique extérieur de sorte que le champ terrestre a dû être compensé.
\end{abstract}

Abstract. - We give the first experimental observation of a magnetic hyperfine structure in the infrared spectrum of ammonia through saturation spectroscopy. The $v_{2}$ as $\mathbf{R}(2,0)$ transition of ${ }^{15} \mathrm{NH}_{3}$ in close coincidence with the $10 \mathrm{R}(42) \mathrm{CO}_{2}$ laser line has been recorded with a $2 \mathrm{kHz}$ halfwidth, which was limited by the molecular transit time across the laser beam. The observed spectrum appeared to be very sensitive to an external magnetic field so that the earth field has to be compensated.

Dans le cadre d'un programme d'étude de structures hyperfines et superfines de nombreuses molécules, nous présentons la première observation expérimentale d'une structure hyperfine magnétique dans la bande $v_{2}$ de la molécule d'ammoniac.

Dans le passé, il a déjà été possible de mettre en évidence la dépendance vibrationnelle de la constante d'interaction hyperfine quadrupolaire électrique dans la bande $v_{2}$ [1] et plus récemment dans la bande $2 v_{2}$ [2] de ${ }^{14} \mathrm{NH}_{3}$. De telles observations nécessitaient un pouvoir de résolution de quelques dizaines de $\mathrm{kHz}$. L'observation d'une structure hyperfine magnétique requiert une résolution de l'ordre du kilohertz $\left(\simeq 3 \times 10^{-8} \mathrm{~cm}^{-1}\right)$.

Cette structure hyperfine magnétique a été observée à l'aide du spectromètre infrarouge de Villetaneuse décrit dans les références [3-6]. Celui-ci est composé essentiellement de deux lasers à $\mathrm{CO}_{2}$, contrôlés en fréquence, associés à deux cuves d'absorption qui permettent d'utiliser les techniques classiques d'absorption saturée.

Le premier laser à $\mathrm{CO}_{2}$, fonctionnant à basse pression, est stabilisé en fréquence sur une raie de saturation moléculaire et sert de référence de fréquence. En particulier, l'asservissement en fréquence avec une raie de la molécule $\mathrm{OsO}_{4}$ comme référence a déjà permis d'obtenir une

$\left(^{*}\right)$ Associé au C.N.R.S., no 282. 
pureté spectrale de l'ordre de $10 \mathrm{~Hz}$ et une stabilité à long terme meilleure que $10^{-13}$ sur des temps typiques d'enregistrement de plusieurs minutes. Le second laser à $\mathrm{CO}_{2}$ est décalé en fréquence par rapport au précédent, à l'aide d'un verrouillage en phase du battement de ces deux lasers sur la fréquence très stable d'un synthétiseur radio-fréquence. Cette boucle d'asservissement permet à la fois de transférer la stabilité à court terme et à long terme du laser de référence au laser décalé et d'effectuer un balayage en fréquence parfaitement linéaire grâce à la commande $\mathrm{du}$ synthétiseur par ordinateur. Le faisceau du laser accordable est alors agrandi à un diamètre $2 w_{0}=8 \mathrm{~cm}$ et envoyé dans une grande cuve d'absorption (longueur $18 \mathrm{~m}$, diamètre $70 \mathrm{~cm}$ ), où il vient sonder l'absorption des molécules sur un trajet de $108 \mathrm{~m}$. Le défaut de planéité des fronts d'ondes est inférieur à $\lambda / 10$ à $10 \mu \mathrm{m}$. A très basse pression $\left(\simeq 10^{-5}\right.$ torr $)$ la largeur de raie n'est plus limitée que par le temps fini d'interaction des molécules avec le champ laser, ce qui permet d'obtenir une demi-largeur de raie à mi-hauteur de $650 \mathrm{~Hz}$ avec une molécule lourde telle que $\mathrm{OsO}_{4}$, mais défavorise une molécule légère telle que $\mathrm{NH}_{3}$. L'élargissement dû au temps de vol des molécules à travers le faisceau s'élève dans ce cas à $1,8 \mathrm{kHz}$ (demi-largeur à mi-hauteur).

Notre choix pour la première mise en évidence de la structure hyperfine magnétique de l'ammoniac a porté sur la transition as $\mathrm{R}(2,0)$ dans la bande $v_{2}$ de ${ }^{15} \mathrm{NH}_{3}$ en quasi-coïncidence avec la raie $10 \mathrm{R}(42)$ du laser à $\mathrm{CO}_{2}$. Nous observons cette raie de ${ }^{15} \mathrm{NH}_{3}$ à $988,6484 \mathrm{~cm}^{-1}$ (en bon accord avec les valeurs données dans les références [7] et [8]; soit à environ $+53 \mathrm{MHz}$ du centre de la raie $\mathbf{R}(42)$ de sorte qu'elle n'est accessible qu'à l'aide d'un laser guide d'ondes dont la plage d'oscillation peut s'étendre sur plusieurs centaines de MHz. Nous n'avons pas cherché, au cours

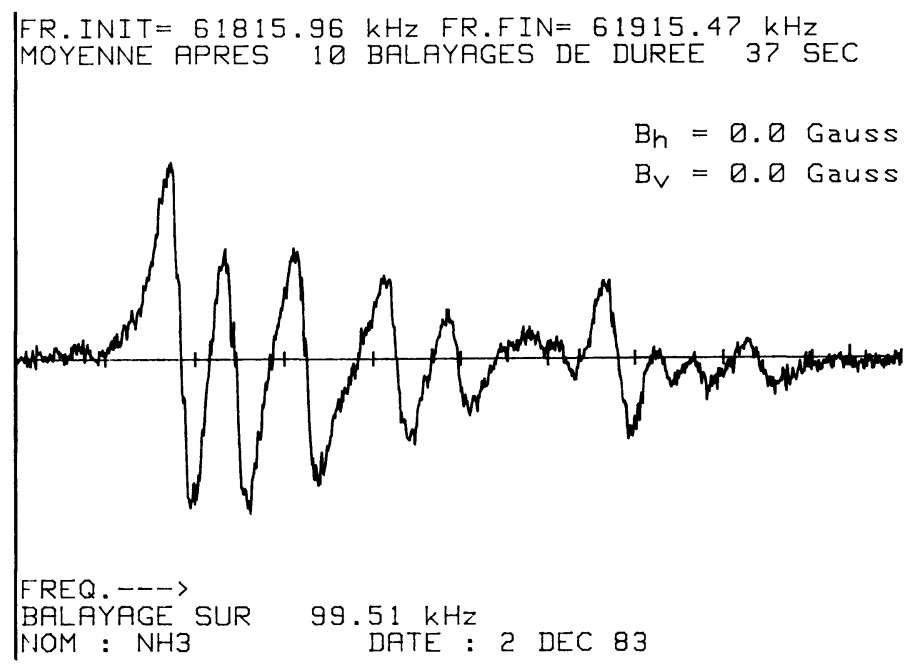

Fig. 1a. - Spectre en dérivée première de la raie as $\mathrm{R}(2,0)$ de la bande $v_{2}$ de ${ }^{15} \mathrm{NH}_{3}$. Fréquence de modulation : $750 \mathrm{~Hz}$; pression : $1,5 \times 10^{-5}$ torr; intensité laser : $3 \mu \mathrm{W}$; constańte de temps : $30 \mathrm{~ms}$. Dix balayages de durée $37 \mathrm{~s}$ ont été additionnés. Le champ magnétique dans la cellule d'absorption est réduit en dessous du dixième de la valeur du champ magnétique terrestre. La largeur de raie $(2,1 \mathrm{kHz} \mathrm{HWHM})$ est essentiellement limitée par le temps de vol des molécules à travers le faisceau de diamètre $2 w_{0}=8 \mathrm{~cm}$. Les élargissements par pression, par saturation et par modulation sont inférieurs à $500 \mathrm{~Hz}$.

[First derivative spectrum of the $v_{2}$ as $\mathrm{R}(2,0)$ line of ${ }^{15} \mathrm{NH}_{3}$. The modulation frequency is $750 \mathrm{~Hz}$. The pressure is $\simeq 1.5 \times 10^{-5}$ torr. The laser power is $3 \mu$ watt. The time constant is $30 \mathrm{~ms}$, and 10 sweeps of $37 \mathrm{~s}$ duration are added. The magnetic field in the cell is reduced below $10 \%$ of the earth magnetic field. The linewidth ( $2.1 \mathrm{kHz}$ HWHM) is mainly limited by the transit time across the $8 \mathrm{~cm}$ diameter laser beam. Pressure broadening, saturation broadening and modulation broadening are below $500 \mathrm{~Hz}$.] 
de cette étude, à mesurer la fréquence absolue de cette raie avec précision, ce qui ne poserait aucun problème en cas de nécessité.

Pour cette expérience sur ${ }^{15} \mathrm{NH}_{3}$, nous avons utilisé comme référence de fréquence une raie de saturation de la molécule $\mathrm{C}_{2} \mathrm{~F}_{2} \mathrm{Cl}_{2}$, présentée sur la figure 2. En dépit du médiocre rapport signal sur bruit obtenu sur cette raie $(200 / \sqrt{\mathrm{Hz}})$ il a été possible d'observer des largeurs de raie sur ${ }^{15} \mathrm{NH}_{3}$ de l'ordre de $2 \mathrm{kHz}(\mathrm{HWHM})$ grâce à l'excellente stabilité intrinsèque du laser de référence.

Etant donné que les 2 composantes de recul sont distantes de $216 \mathrm{~Hz}$, il est remarquable d'observer une largeur de raie aussi proche de la limitation imposée par le temps de vol. C'est là une manifestation de l'affinement des raies dû à la contribution anormale des molécules de vitesse lente au signal d'absorption saturée [9].

La figure la présente le spectre obtenu à la plus haute résolution, avec une pression dans la cuve d'absorption de $1.5 \times 10^{-5}$ torr. L'ensemble de la structure s'étend sur une centaine de kilohertz. La reproductibilité des positions relatives des diverses résonances était meilleure que la centaine de hertz d'un enregistrement à l'autre. Nous avons noté une grande sensibilité du spectre au champ magnétique terrestre, aisement expliquée par les valeurs élevées des facteurs de Landé, conduisant à un effet Zeeman résiduel. Celui-ci est manifeste sur la figure $1 \mathrm{~b}$ où le champ terrestre n'est pas compensé, tandis que la compensation du champ magnétique terrestre est réalisée à mieux que $10 \%$ dans le cas de la figure la. La composante verticale, $\mathbf{B}_{\mathbf{v}}$, est annulée par le champ créé par le courant de 2 bobines de Helmholtz. L'orientation Nord-Sud de la cuve d'absorption a permis une compensation de la composante horizontale, $\mathbf{B}_{h}$, grâce à un courant parcourant un solénoïde coaxial. Enfin, la figure 1c montre encore plus clairement l'influence d'un champ magnétique sur la structure : on observe une très nette réduction de l'intensité et de la résolution des composantes dans le cas où règne un champ de $3 \mathbf{B}_{\mathbf{v}}$ dans la cuve d'absorption.

L'interprétation théorique de cette structure hyperfine magnétique est en cours et a été considérablement facilitée par les nombreux travaux effectués sur l'état vibrationnel fondamental de l'ammoniac [10-12].

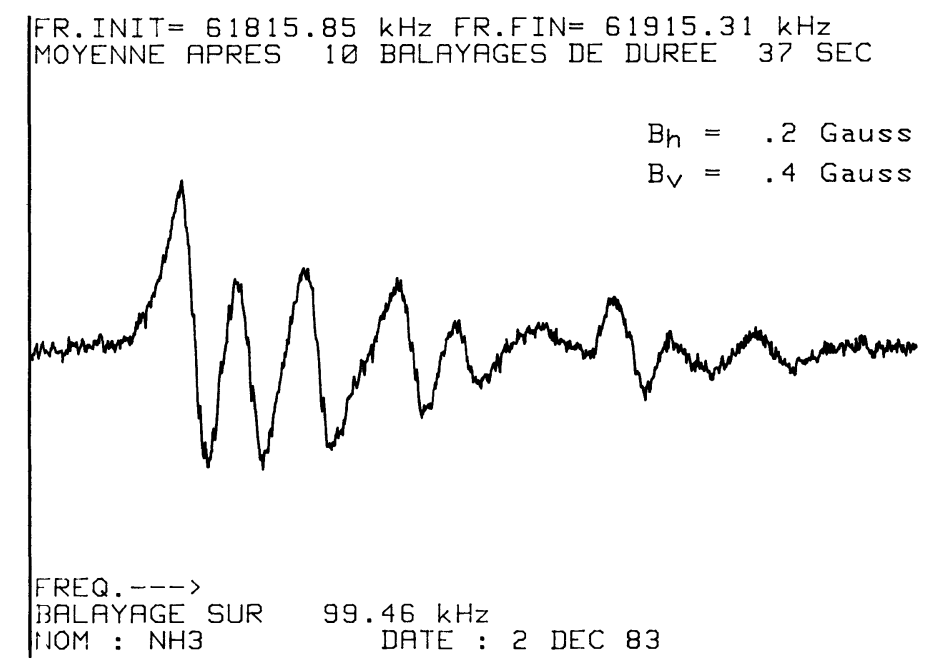

Fig. 1b. - Mêmes conditions que figure 1a, mais le champ magnétique terrestre n'est pas compensé. Noter l'élargissement des résonances, plus marqué sur la droite du spectre.

[Same as figure 1a. The magnetic field in the cell is equal to the earth magnetic field. Note the larger broadening of the lines on the right of the spectrum.] 


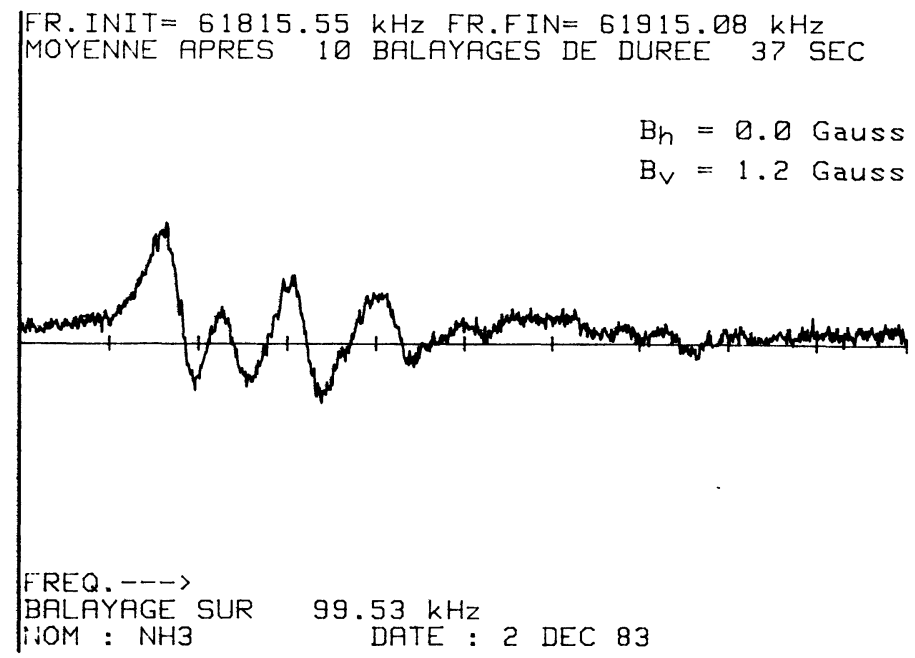

Fig. 1c. - Mêmes conditions que figure 1a. Le champ magnétique dans la cellule est vertical et vaut 3 fois la composante verticale du champ terrestre.

[Same as figure la. The magnetic field in the cell is vertical and is equal to 3 times the vertical component of the earth magnetic field.]

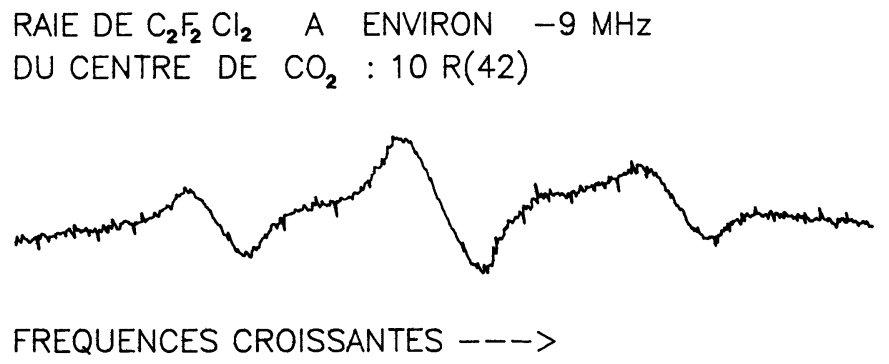

Fig. 2. - Spectre en dérivée première de la raie d'absorption de la molécule $\mathrm{C}_{2} \mathrm{~F}_{2} \mathrm{Cl}_{2}$ utilisée pour stabiliser la fréquence du laser de référence. Cette raie, située à $988,6463 \mathrm{~cm}^{-1}$, est enregistrée à l'aide d'un laser à $\mathrm{CO}_{2}$ libre, avec une pression de $2 \times 10^{-3}$ torr dans la cuve d'absorption. Constante de temps : $30 \mathrm{~ms}$. La structure s'étend sur environ $500 \mathrm{kHz}$ et le laser de référence était verrouillé sur la composante centrale de ce triplet à l'aide d'une boucle d'asservissement utilisant l'harmonique 3 du signal de saturation modulé à $18 \mathrm{kHz}$.

[First derivative spectrum of the $\mathrm{C}_{2} \mathrm{~F}_{2} \mathrm{Cl}_{2}$ reference line recorded with a free-running conventional $\mathrm{CO}_{2}$ laser in the large absorption cell at a $2 \times 10^{-3}$ torr pressure, at $988.6463 \mathrm{~cm}^{-1}$. The time constant is $30 \mathrm{~ms}$. The structure extends over approximately $500 \mathrm{kHz}$. The reference laser was frequency-locked to the central peak of this triplet with a servo-loop using the third harmonic $(3 f=54 \mathrm{kHz})$ of the frequency-modulated saturation signal.]

Un bon accord avec le spectre expérimental a d'ores et déjà été obtenu [13] avec une matrice hamiltonienne dont certains facteurs de phase ont dû être corrigés par rapport à celle utilisée par Kukolich [10].

Enfin une étude plus exhaustive des structures hyperfines de la bande $v_{2}$ de ${ }^{15} \mathrm{NH}_{3}$ et ${ }^{14} \mathrm{NH}_{3}$ est actuellement poursuivie grâce à d'autres coïncidences de raies d'absorption de ces molécules avec les raies laser de $\mathrm{CO}_{2}$ et $\mathrm{N}_{2} \mathrm{O}$. 


\section{Bibliographie}

[1] Ouhayoun, M., Bordé, Ch. J. et BordÉ, J., Mol. Phys. 33 (1977) 597.

[2] Weber, W. H. et Terhune, R. W., J. Chem. Phys. 78 (1983) 6422.

[3] Bordé, Ch. J., Ouhayoun, M., Van lerberghe, A., Salomon. Ch., Avrillier, S., Cantrell, C. D. et BordÉ, J., Laser Spectroscopy IV, édité par H. Walther et K. W. Rothe (Springer Verlag) 1979 , p. 142-153.

[4] Salomon, Ch., Bréant, Ch., Bordé, Ch. J. et Barger, R. L., J. Physique Colloq. 42 (1981) C8-3.

Clairon, A., Van Lerberghe, A., Bréant, Ch., Salomon, Ch., Camy, G. et Borde, Ch. J., J. Physique Colloq. 42 (1981) C8-127.

[5] BordÉ, Ch. J., Rev. du Cethedec. Ondes et signal, NS 83-1 (1983) 1-118.

[6] Salomon, Ch., Bréant, Ch., Van lerberghe, A., Camy, G. et Bordé, Ch. J., Appl. Phys. B 29 (1982) 153.

[7] Job, V. A., Patel, N. D., D'Cunha, R. et Kartha, V. B., J. Mol. Spectrosc. 101 (1983) 48.

[8] Henry, L., Valentin, A., D'Cunha, R., Urban, S. et Narahari RaO, K., 39th Symposium on Molecular Spectroscopy, Columbus (1984).

[9] Borde, Ch. J., Hall, J. L., Kunasz, C. V., Hummer, D. G., Phys. Rev. A 14 (1976) 263.

[10] Kuxolich, S. G., Phys. Rev. 156 (1967) 83.

[11] Kuxolich, S. G., Phys. Rev. 172 (1968) 59.

[12] Hougen, J. T., J. Chem. Phys. 57 (1972) 4207.

[13] Bordé, Ch. J., Chardonnet, Ch. et BordÉ, J., à paraître. 